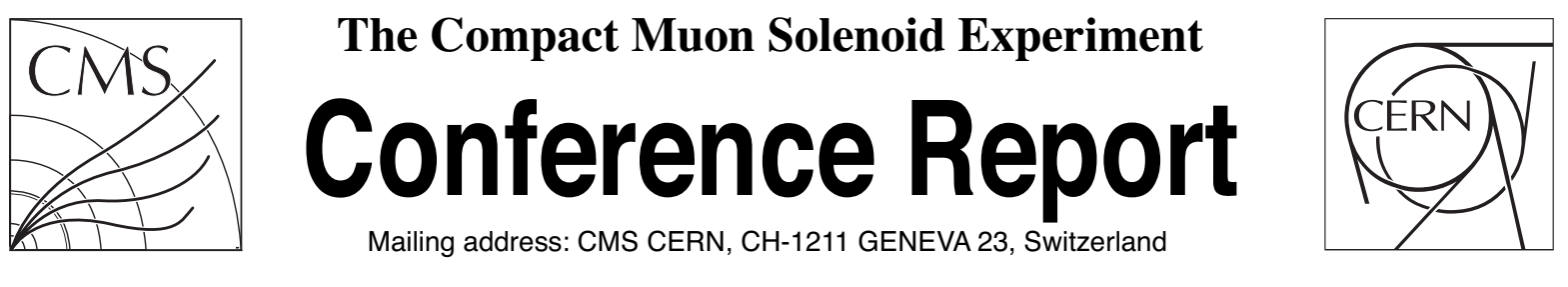

03 April 2013 (v2, 16 April 2013)

\title{
Planar silicon sensors for the CMS Tracker upgrade
}

\author{
Alexandra Junkes for the CMS Collaboration
}

\begin{abstract}
The CMS tracker collaboration has initiated a large material investigation and irradiation campaign to identify the silicon material and design that fulfills all requirements for detectors for the highluminosity phase of the Large Hadron Collider (HL-LHC).A variety of silicon p-in-n and n-in-p testsensors made from Float Zone, Deep-Diffused FZ and Magnetic Czochralski materials were manufactured by one single industrial producer, thus guaranteeing similar conditions for the production and design of the test-structures. Properties of different silicon materials and design choices have been systematically studied and compared.The samples have been irradiated with $1 \mathrm{MeV}$ neutrons and protons corresponding to maximal fluences as expected for the positions of detector layers in the future tracker. Irradiations with protons of different energies $(23 \mathrm{MeV}$ and $23 \mathrm{GeV})$ have been performed to evaluate the energy dependence of the defect generation in oxygen rich material. All materials have been characterized before and after irradiations, and throughout an annealing treatment. The measurements performed on the structures include electrical sensor characterization, measurement of the collected charge injected with a beta source and laser light and bulk defect characterization. In this paper, results from the ongoing campaign are presented.
\end{abstract}




\title{
Planar silicon sensors for the CMS tracker upgrade
}

\author{
Alexandra Junkes on behalf of the CMS Tracker Collaboration* \\ Brown University (USA) and Hamburg University (Germany)
}

\begin{abstract}
The CMS tracker collaboration has initiated a large material investigation and irradiation campaign to identify the silicon material and design that fulfills all requirements for detectors for the high-luminosity phase of the Large Hadron Collider (HL-LHC).

A variety of silicon $p$-in- $n$ and $n$-in- $p$ test-sensors made from Float Zone, Deep-Diffused FZ and Magnetic Czochralski materials were manufactured by one single industrial producer, thus guaranteeing similar conditions for the production and design of the test-structures. Properties of different silicon materials and design choices have been systematically studied and compared.

The samples have been irradiated with neutrons $(1 \mathrm{MeV})$ and protons $(23 \mathrm{MeV}$ and $23 \mathrm{GeV})$ corresponding to maximal fluences as expected for the positions of detector layers in the future tracker. Irradiations with protons of different energies have been performed to evaluate the energy dependence of the defect generation in oxygen rich material. All materials have been characterized before and after irradiations, and throughout an annealing treatment. The measurements performed on the structures include electrical sensor characterization, measurement of the collected charge injected with a beta source or laser light and bulk defect characterization. In this paper, a selection of results from the ongoing studies are presented.
\end{abstract}

Keywords: Radiation damage, Silicon sensors, Tracking detectors, CMS, Material characterisation

\section{Introduction}

The foreseen upgrade to the high-luminosity phase of the Large Hadron Collider (HL-LHC) is expected to result in a further increase of the luminosity to $5 \times 10^{34} \mathrm{~s}^{-1} \mathrm{~cm}^{-2}$ with a total of $3000 \mathrm{fb}^{-1}$ of collosions [1]. This leads to exceptional demands for the experiments located at the LHC. The detectors have to withstand a harsh radiation exposure (up to $\Phi_{e q}=2 \times 10^{16} \mathrm{~cm}^{-2}$ ) as well as an increased particle density. Hence, all experiments are preparing upgrades of the detectors for the high-luminosity phase.

Currently, more than $200 \mathrm{~m}^{2}$ of planar silicon sensors are used in the CMS experiment. This technology has proven to be very reliable and predictable and is considered to be the best choice for the outer regions of a future CMS tracker. However, new silicon materials and production processes offer further potential for optimization.

In the recent years, multiple approaches have been started to find the optimal material and sensor design [2]. Test-sensors from different producers with deviating specifications are often hard to compare because of their varying starting-conditions. The CMS tracker collaboration has therefore initiated a comprehensive sensor measurement campaign to systematically study and compare different silicon materials, production processes and sensor designs under identical conditions. A realistic irradiation environment can be provided by irradiations with particle types and fluences according to simulations of the expected fluences in the CMS detector at a position in the detector (more information about expected radiation exposure can be

*Corresponding author: alexandra.junkes@ desy.de found in [3]).

Important parameters for the operation of the detector are the increase of the radiation-induced current $\Delta I$, the depletion voltage $V_{f d}$ and the collected charge. The radiation-induced current is known to be a universal parameter, following the fluence with $\Delta I=\alpha \Phi_{e q} V$ where $\alpha$ is the current related damage rate, $\Phi_{e q}$ the $1 \mathrm{MeV}$ neutron equivalent fluence $\left(\Phi_{e q}=\Phi_{\text {particle }} \cdot \kappa, \kappa\right.$ being the hardness factor of the respective particle) and $V$ the volume of the pad diode [4]. $V_{f d}$ changes with irradiation, depending on material and particle type. Thus the choice of the material has a large impact on the operational conditions of the tracker. $V_{f d}$ is connected to the effective doping concentration $N_{e f f}$ by

$$
V_{f d}=\frac{q_{0}}{2 \epsilon \epsilon_{0}}\left|N_{e f f}\right| d^{2},
$$

with $q_{0}$ being the elementary charge, $d$ the thickness of the active area and $\epsilon \epsilon_{0}$ the permittivity of silicon.

The charge collected from a sensor is the most important parameter for the applicability of sensors in the future tracker. The combination of radiation effects (eg. change of $V_{f d}, I_{f d}$ and trapping) lead to a reduction of the induced signal that has to be far away from the noise threshold.

Given the requirements towards the future outer tracker, this campaign seeks to identify the material and design with lowest depletion voltage, low currents and highest signal-to-noise ratio after irradiation. Additionally, there is a strong demand for having a cost-efficient solution with a low material budget. 


\section{Materials, irradiations and measurement techniques}

\section{Materials and structures}

Sensors were produced by one single industrial producer (Hamamatsu Photonics K.K.) to guarantee similar conditions for all materials, designs and production technologies and at the same time fulfill the demands towards high quality and quantity.

Since charge trapping and current generation increase independent of the material with the fluence, the only parameter that can be optimized by the choice of the material is $V_{f d}$ (and by this also $N_{e f f}$ ). Moreover, this parameter has a major impact on the change of the electric field distribution with irradiation in the sensor and therefore the overall performance of the device. The main difference between silicon materials is the oxygen content of the material, which is predominantly introduced during the growth process of the silicon ingot. Therefore, materials produced with different growth techniques were selected for the analysis of candidate materials. Standard Float Zone (FZ) and Magnetic Czochralski (MCz) materials of $200 \mu \mathrm{m}$ thickness and Deep-Diffused FZ (dd-FZ) with three different active thicknesses were produced in $p$-in- $n$ as well as $n$-in- $p$ technology, using two different strip isolation techniques for the $n$-in- $p$ materials ( $p$-spray and $p$-stop). All dd-FZ sensors were produced from high resistivity FZ with a wafer thickness of $320 \mu \mathrm{m}$. Effective active layer thicknesses of $120 \mu \mathrm{m}, 200 \mu \mathrm{m}$ and $290 \mu \mathrm{m}$ were achieved by deep-diffusion of dopants from the rear side. The oxygen concentration [O] of the materials measured by Secondary Ion Mass Spectroscopy (SIMS) is presented in Fig. 1. It should be noted that the oxygen content in the dd-FZ sensors is much higher than expected for FZ wafers. This is a result of the high-temperature deep-diffusion process, leading to a diffusion of oxygen from the $\mathrm{SiO}_{i}$ surface layer into the bulk material.

Thin sensors are known to show a smaller degradation of the collected charge with increasing fluence compared to thick ones [2]. Less thick sensor material would also help to reduce the material budget of the tracking detector. Therefore test-sensors were produced in various thicknesses.

A variety of test-structures, planar diodes, fully functional strip and pixel sensors was manufactured for investigating the electrical characteristics and the impact of radiation damage on the electrical properties and on the collected charge.

\section{Irradiations}

An irradiation scenario with mixed particle irradiations was defined to test the radiation damage as expected for the sensor layers in the outer tracker. The sensors were irradiated with protons or neutrons and then characterized electrically to extract the radiation damage inflicted by the respective particle type. Then the irradiation with the other particle type was carried out on the same sensors and the accumulated damage was characterized. Additionally a sub-set of sensors was annealed and analyzed further after only one type of irradiation. This procedure allows to understand the radiation damage by mixed irradiation, using the combined information from the damage inflicted by each particle type.

Reactor neutron $(\kappa=0.91)$ irradiations have been carried out

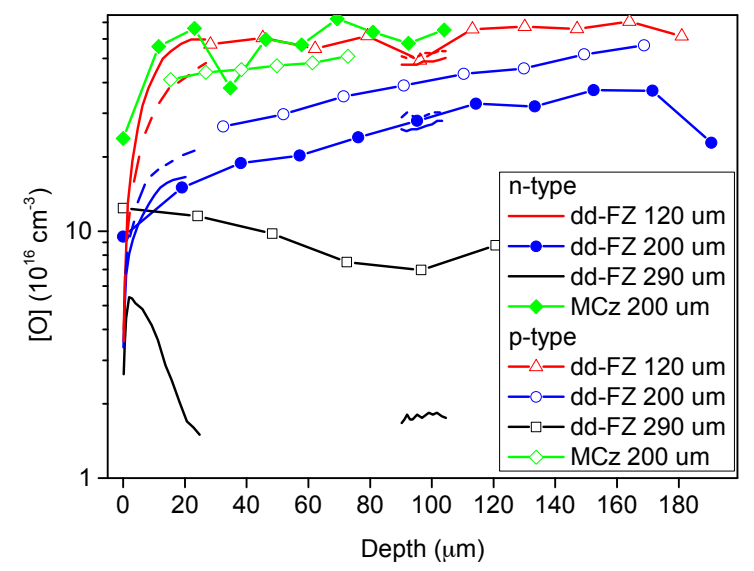

Figure 1: Oxygen concentration measured for two sets of dd$\mathrm{FZ}$ and $\mathrm{MCz}$ sensors. Continuous lines denote the first set and results presented with symbols mark the second set.

in the TRIGA Mark II reactor ${ }^{1}$ at Ljubljana, Slovenia. In order to understand the impact of different proton energies of the radiation damage, protons from two facilities were chosen: $23 \mathrm{MeV}$ protons $(\kappa=2.00)$ from $\mathrm{ZAG}^{2}$, Karlsruhe, Germany and $23 \mathrm{GeV}(\kappa=0.62)$ proton irradiations from the Proton Synchrotron PS at CERN ${ }^{3}$.

\section{Measurement techniques}

Current-Voltage $(I V)$ and Capacitance-Voltage $(C V)$ measurements were performed on pad diodes for obtaining the full depletion voltage $\left(V_{f d}\right)$ and the current at full depletion $\left(I_{f d}\right)$ and at possible maximum operation voltages $(600 \mathrm{~V}, 900 \mathrm{~V})$. $V_{f d}$ is evaluated from the intersect of two linear fits to the $1 / C^{2}-V$ curve and $I_{f d}$ is extracted at a voltage of $1.05 \cdot V_{f d}$ to account for the broad transition region between the linear part of $1 / C^{2}-V$ and the onset of saturation resulting from the deep-diffusion process.

Due to the increase of current with irradiation that exceeds the specifications of the measurement setups, sensors have to be cooled to $0{ }^{\circ} \mathrm{C}$ or $-20^{\circ} \mathrm{C}$. Current values presented in this work are scaled to $20^{\circ} \mathrm{C}$ using [5]. Moreover, radiation-induced deep trap levels lead to a frequency and temperature dependence of the capacitance. This has to be taken into account by choosing the best suited frequency for the $\mathrm{CV}$ measurement. Other studies have analyzed the optimal frequency dependent on the measurement temperature [6]. However, for high fluences $\left(\Phi_{e q} \gtrsim 1 \times 10^{15} \mathrm{~cm}^{-2}\right.$ ) further studies on the extraction of $V_{f d}$ and the frequency dependence are needed. Measurements presented here were performed at $455 \mathrm{~Hz}$ for measurements at $-20^{\circ} \mathrm{C}, 1 \mathrm{kHz}$ for $0{ }^{\circ} \mathrm{C}$ and $10 \mathrm{kHz}$ for $20^{\circ} \mathrm{C}$ (see also [7]).

\footnotetext{
${ }^{1}$ http://www.rcp.ijs.si/ric/index-a.htm

${ }^{2}$ http://www.zyklotron-ag.de

${ }^{3}$ https://irradiation.web.cern.ch/irradiation/irrad1.htm
} 


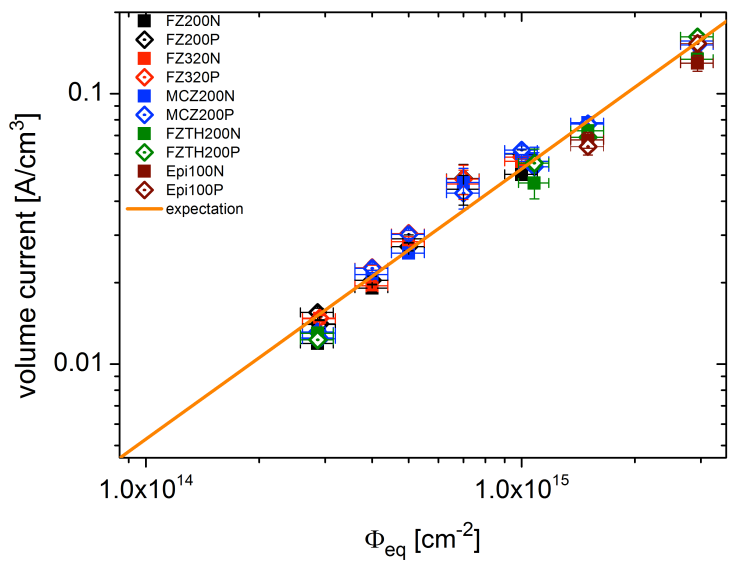

Figure 2: Volume current at $V_{\text {bias }}=1.05 \cdot V_{f d}$ measured on pad diodes.

\section{Results}

\section{Radiation-induced current}

The current at full depletion $\left(V=1.05 \cdot V_{f d}\right)$ measured for pad diodes is presented in Fig. 2 for a fluence range of $\Phi_{e q}=10^{14} \mathrm{~cm}^{-2}$ to $\Phi_{e q}=3 \times 10^{15} \mathrm{~cm}^{-2}$. The orange straight line is the expectation for $\Delta I / V$ taken from [4] and the slope represents $\alpha$. The sensors are of different type and material, and were irradiated with neutrons, $23 \mathrm{GeV}$ protons and $23 \mathrm{MeV}$ protons. Measurements were done after 10 minutes at $60^{\circ} \mathrm{C}$ or, in case sensors annealed during irradiations, were scaled to the same annealing step by using [4].

The volume current measurements on strip sensors is presented in Fig. 3. The thickness of the device was obtained from measurements on pad diodes of similar material and is used to calculate the volume of the strip sensor. The current was taken at a bias voltage of $1.2 \cdot V_{f d}$ or $600 \mathrm{~V}$ (the limit of the present CMS tracker) for sensors with $V_{f d}$ larger than $600 \mathrm{~V}$. As a result, several sensors are not fully depleted. As for the case of the pad diodes, the expected current value calculated from [4] is presented as a straight line in Fig. 3. The total current of the

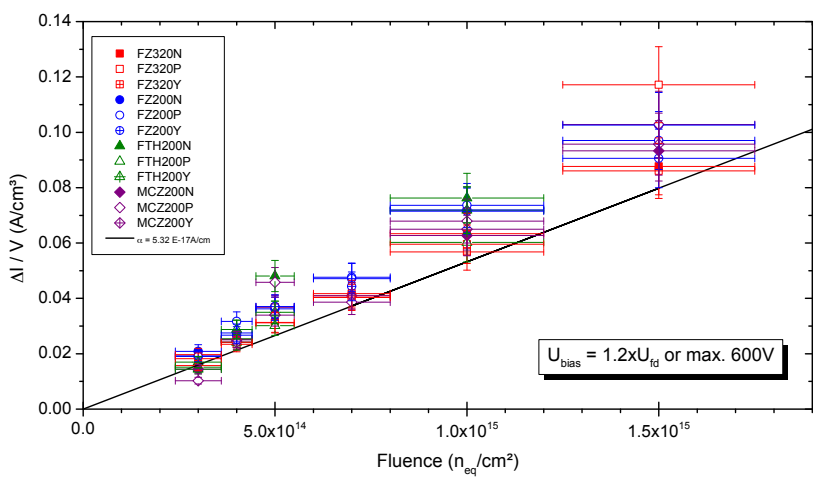

Figure 3: Volume current at $V_{\text {bias }}=1.2 \cdot V_{f d}$ or $600 \mathrm{~V}$ (see text) measured on strip sensors. Measurements performed at $-20{ }^{\circ} \mathrm{C}$, annealed at $60^{\circ} \mathrm{C}$ for 10 minutes.

strip sensors exceeds the expectations from pad diode measure- ments.

Material dependence of irradiated pad diodes and influence of particle energy on $N_{\text {eff }}$

Fig. 4 presents the absolute value of $N_{\text {eff }}$ (left axis) and $V_{f d}$ (right axis) for $23 \mathrm{MeV}$ proton irradiated diodes up to a fluence of $\Phi_{e q}=1 \times 10^{15} \mathrm{~cm}^{-2}$ for the annealing step of 10 minutes at $60{ }^{\circ} \mathrm{C}$. $V_{f d}$ values were scaled to an active thickness of $200 \mu \mathrm{m}$ according to Eq. (1). The materials presented here are dd-FZ with thicknesses of $290 \mu \mathrm{m}$ (red triangles) and $220 \mu \mathrm{m}$ (black squares) and $\mathrm{MCz}$ with a thickness of $200 \mu \mathrm{m}$ (blue circles). Continuous lines represent $n$-type sensors while dashed lines denote p-type sensors.

$V_{f d}$ of p-type sensors is higher than for corresponding $n$-type

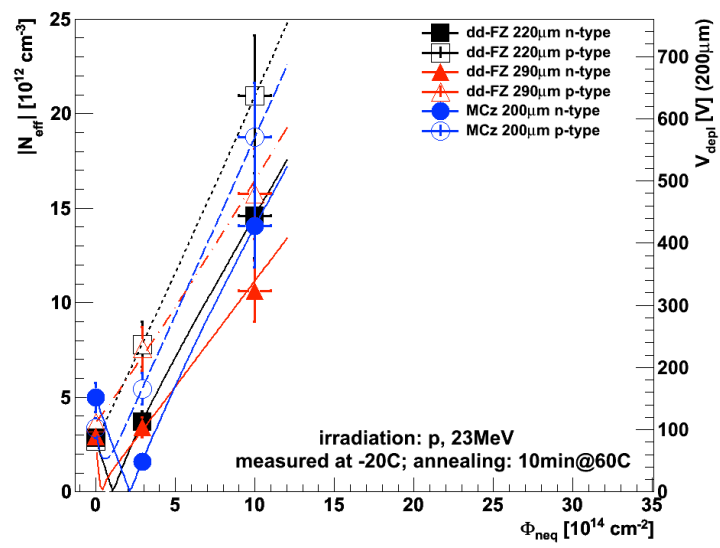

Figure 4: $N_{e f f}$ and $V_{f d}$ for $23 \mathrm{MeV}$ irradiated pad diodes with varying oxygen content. Annealing step at 10 minutes at $60^{\circ} \mathrm{C}$.

sensors and the sign of the space charge remains negative in these materials. All n-type sensors undergo "type inversion", which means that the n-bulk becomes $p$-type-like and the effective $p n$-junction is on the rear side at the former $n-n^{++}$junction. However, the electric field distribution in those sensors is much more complicated than discussed here because of the formation of a double junction effect (for more information see [9, 10]). Fig. 5 presents $N_{e f f}$ (left axis) and $V_{f d}$ (right axis, scaled to $200 \mu \mathrm{m}$ ) for $23 \mathrm{GeV}$ proton irradiated pad diodes up to a fluence of $\Phi_{e q}=3 \times 10^{15} \mathrm{~cm}^{-2}$. The materials presented here are ddFZ with thicknesses of $220 \mu \mathrm{m}$ (black squares) and FZ with a thickness of $200 \mu \mathrm{m}$ (green triangles) and MCz with a thickness of $200 \mu \mathrm{m}$ (blue circles). Continuous lines in Fig. 5 represent $n$-type sensors whereas p-type sensors are denoted by dashed lines.

As a result of the long exposure time during the irradiations with $23 \mathrm{GeV}$ protons, non-negligible annealing takes place especially for high fluence values. Because of this, fluence steps presented in Fig. 5 are at different annealing stages. For fluences up to $\Phi_{e q}=3 \times 10^{14} \mathrm{~cm}^{-2}$ the annealing step is $10 \mathrm{~min}$ utes at $60^{\circ} \mathrm{C}$, for higher fluence values the annealing time increases up to 80 minutes at $60^{\circ} \mathrm{C}$.

The dd-FZ has been irradiated only up to a fluence of $\Phi_{e q}=3 \times 10^{14} \mathrm{~cm}^{-2}$, therefore it is not possible to estimate the slope of the dependence of $N_{e f f}$ on the dose for higher fluences. 


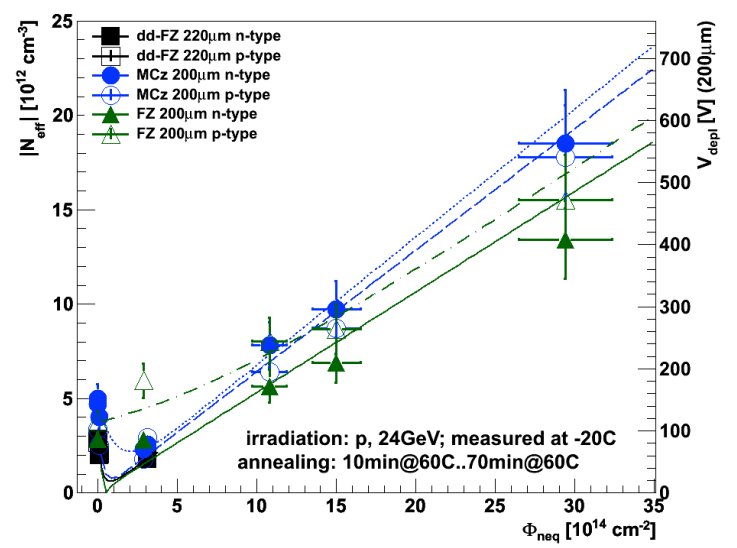

Figure 5: $N_{e f f}$ and $V_{f d}$ for pad diodes with different oxygen content and irradiated with $23 \mathrm{GeV}$ protons. Annealing see text.

However, it can be observed that $V_{f d}$ is higher for the FZ p-type material, but lower for the $\mathrm{MCz}$ samples. This can be explained by the generation of donors in oxygen rich material after 23 $\mathrm{GeV}$ proton irradiation.

In contrast to the $23 \mathrm{MeV}$ proton irradiated materials, none of the n-type materials undergoes type inversion, with the FZ $200 \mu \mathrm{m}$ being at the borderline between type inversion and nontype inversion. For the oxygen rich materials dd-FZ and $\mathrm{MCz}$ no type inversion could be observed. A detailed analysis of TCT measurements addressing the sign of the space charge can be found in [11].

A comparison of $V_{f d}$ for $23 \mathrm{GeV}$ and $23 \mathrm{MeV}$ proton irradiated sensors reveals a much steeper increase of $V_{f d}$ as function of $\Phi$ the samples irradiated with $23 \mathrm{MeV}$ protons. However, this might be misleading, since the annealing time for the sensors is not similar and $V_{f d}$ is supposed to decrease especially for the MeV-irradiated sensors.

\section{Mixed irradiations}

As seen in [12] the mixed irradiation of $23 \mathrm{GeV}$ protons followed by $1 \mathrm{MeV}$ neutron irradiation was expected to yield a smaller increase of $V_{f d}$ compared to the proton irradiated only case (compensation) for oxygen rich material. Fig. 6 presents $N_{\text {eff }}$ (left axis) and $V_{f d}$ (right axis, scaled to $200 \mu \mathrm{m}$ ) after irradiation with $23 \mathrm{MeV}$ protons and additional neutron irradiation. The expected compensation effect cannot be observed. The resulting $V_{f d}$ from mixed irradiations is similar to the accumulated $V_{f d}$ from irradiations with only one type of particle.

\section{Conclusions}

Radiation-induced currents were compared for pad diodes and strip sensors made of similar materials. The total current of strip sensors exceeds the expectation from pad diodes possibly due to contributions from the $\mathrm{SiO}_{2}-\mathrm{Si}$ interface between the strips or high electric fields at the border of the strip or $p$-stop implants.

A comparison of $V_{f d}$ was evaluated for oxygen rich materials

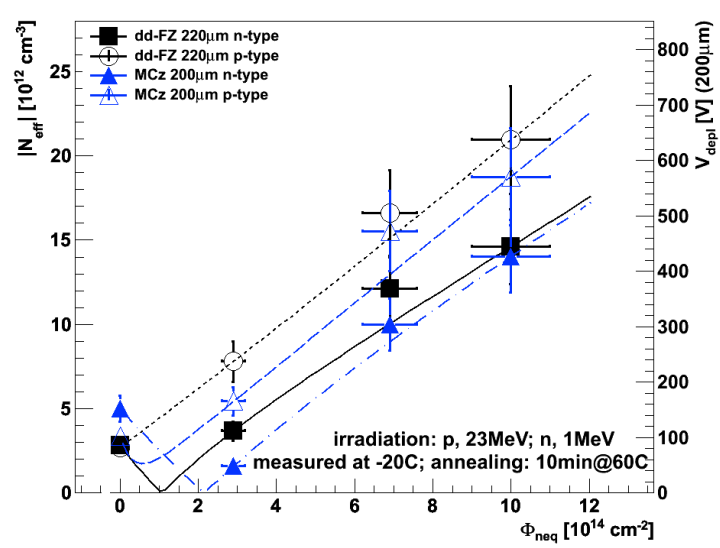

Figure 6: $N_{e f f}$ and $V_{f d}$ for sole $23 \mathrm{MeV}$ proton irradiations and one mixed proton and $1 \mathrm{MeV}$ neutron irradiation step (at $\Phi_{e q}=7 \times 10^{14} \mathrm{~cm}^{-2}$ for FZ and MCz materials).

(MCz $200 \mu \mathrm{m}$, dd-FZ $200 \mu \mathrm{m}$ ) and oxygen lean materials (ddFZ $290 \mu \mathrm{m}$, FZ $200 \mu \mathrm{m}$ ) after $23 \mathrm{GeV}$ proton and $23 \mathrm{MeV}$ proton irradiations. After $23 \mathrm{MeV}$ proton irradiations type inversion of all n-type sensors and the consistently higher $V_{f d}$ for p-type sensors point to a high generation of acceptors, nearly independent of the material. On the other hand, oxygen rich n-type sensors (MCz, dd-FZ $200 \mu \mathrm{m})$ do not type invert after irradiations with $23 \mathrm{GeV}$ protons (compare [2]). This can be attributed to a high introduction of donors in oxygen rich materials after high-energy proton irradiations. The donor generation is less pronounced for oxygen lean material (FZ $200 \mu \mathrm{m}$ ) after $23 \mathrm{GeV}$ proton irradiations and consequently $V_{f d}$ of $p$-type samples is higher than of n-type samples.

It was expected to observe a reduction of $V_{f d}$ for two consecutive irradiations with neutrons and protons in oxygen rich materials, as seen in [12]. However, $V_{f d}$ is not reduced for sensors irradiated with $23 \mathrm{MeV}$ protons followed by additional neutron irradiations, compared to the accumulated $V_{f d}$ obtained from the irradiations with only one type of particle. The compensation effect in oxygen rich n-type sensors is a result of the donor generation after $23 \mathrm{GeV}$ proton irradiations, that cannot be observed after $23 \mathrm{MeV}$ proton irradiations.

\section{Acknowledgments}

The research leading to these results has received funding from the European Commission under the FP7 Research Infrastructures project AIDA, grant agreement no. 262025 and the Helmholtz Alliance: Physics at the Terascale.

[1] E. Shaposhnikova, CERN-AB-2008-065 (2008).

[2] The RD50 Collaboration, CMS-CR-2011-308 (2011).

[3] S. Müller, 2010, CMS-TS-2010-042.

[4] M. Moll, PhD thesis, University of Hamburg, 1999

[5] A. Chilingarov, RD50 Workshop, 2004.

[6] M.K. Petterson et. al., NIM A 583 (2007) 189 - 194.

[7] V. Khomenkov, RD50 Workshop, Freiburg, 2009.

[8] R. Marco-Hernández et al., EEE Trans. Nucl. Sci. 56(3), 2009, pp. 1642.

[9] Z. Li, H. W. Kraner, Journal of Electronic Materials, 21(7):701-705, 1992. 
[10] V. Eremin, E. Verbitskaya and Z. Li, NIM A 476 (2002) 556 - 564.

[11] C. Neubüser, Master thesis, University of Hamburg, 2013.

[12] G. Kramberger, V. Cindro, I. Dolenc, I. Mandić, M. Mikuž, M. Zavrtanik, NIM A 609 (2009) $142-148$. 